\title{
Diagnóstico de Fallas en Máquinas Eléctricas Rotatorias Utilizando la Técnica de Espectros de Frecuencia de Bandas Laterales
}

\author{
Roberto Flores y Tomás I. Asiaín \\ Departamento de Ingeniería Eléctrica, SEPI-ESIME-IPN, México D.F., México. \\ (e-mail: robertf_@msn.com, yeriak1@gmail.com)
}

Recibido Oct. 19, 2010; Aceptado Dic. 13, 2010; Versión Final recibida Ene. 28, 2011

\begin{abstract}
Resumen
En el presente trabajo se muestra y se aplica una metodología para el diagnóstico de fallas en máquinas eléctricas rotatorias utilizando la técnica de espectros de frecuencia de bandas laterales de las corrientes de fase. Se establecen sistemas de prueba de laboratorio para verificar las magnitudes de las bandas originadas por fallas tanto mecánicas como eléctricas. Se muestra una serie de resultados para un grupo motor de corriente directa-generador síncrono de 5 kVA desalineado y de dos motores de inducción de 5 HP tipo jaula de ardilla, uno con holgura en soporte de baleros y otro con barras rotas. Además, se comparan los resultados de los espectros empleando un sensor de corriente convencional contra una bobina de Rogowski, obteniendo resultados adecuados para detectar la presencia de falla en las máquinas.
\end{abstract} inducción.

\section{Fault Diagnosis in Rotating Electrical Machines by Side Bands Frequency Spectra Technique}

\begin{abstract}
In this article a methodology to diagnose faults in rotating electrical machines is presented. The method uses spectral frequency analysis of the phase currents sidebands. Online systems to verify the magnitude of these frequencies, caused not only by mechanical faults but also electrical faults, are presented. The results of a faulty set formed by a direct current motor plus a synchronous generator of $5 \mathrm{kVA}$ and two squirrel-cage induction motors of $5 \mathrm{HP}$, one with slacked bearings faults and other with broken rotor bars, are presented. Also, the results of the spectra using a conventional current sensor are compared with those of a Rogowski coil, showing that the results of both sensors are suitable to detect machine faults.
\end{abstract}

Keywords: spectral analysis, sidebands, faults, synchronous generator, induction motor 


\section{INTRODUCCION}

En las máquinas rotatorias, se generan voltajes en los devanados o grupos de bobinas al girar estos de manera mecánica a través de un campo magnético. También, al girar mecánicamente un campo magnético por el devanado o al diseñar un circuito magnético de manera que la reluctancia varíe con la rotación del rotor (Fitzgerald et al., 2004). En general cualquier máquina rotatoria consta de un estator y un rotor separados por un entrehierro, el cual es de vital importancia en las interacciones magnéticas entre estas partes que dan lugar a los voltajes antes mencionados. Sin embargo, aún en máquinas rotatorias nuevas existen pequeñas desviaciones entre la posición que debe guardar el rotor con respecto al estator, lo que origina que se modifique el entrehierro de la máquina y por ende afecte los voltajes inducidos. Debido al desgaste natural de baleros, soportes, al tipo de carga o al acoplamiento mecánico de la máquina se incrementa el desajuste entre el rotor y el estator. Este fenómeno que puede implicar excentricidad, desalineación, entre otros problemas repercute en el desempeño de la máquina (Fitzgerald et al., 2004 y Albino, 2004).

Con el fin de garantizar que la máquina eléctrica rotatoria como tal este operando en forma continua, es necesario contar con técnicas de medición que además de permitir detectar la falla no impliquen el sacarla de operación. Una técnica de medición en línea adecuada para este fin es el análisis espectral de corriente medida a través de la transformada rápida de Fourier (FFT) (Fernández et al., 2000) contenida en los equipos de medición que tienen esta función. Esta técnica de la bandas laterales en el diagnóstico de fallas en máquinas rotatorias tiene varias décadas, pero toma fuerza en los 80's hasta inicios de los 90's y toma forma final en los últimos quince años ya que en la actualidad esta bien reconocida en el ámbito internacional y solo se han presentados cambios significativos en los sensores de corriente y tensión así como en los equipos de registro, ya que han sido utilizados osciloscopios, analizadores de espectros y finalmente la computadora digital con tarjetas de adquisición de datos A/D y programas de computo comerciales o elaborados por los investigadores.

Como antecedentes de esta técnica en forma general se puede mencionar que en los años ochenta se presentó una análisis en estado permanente de motores trifásicos jaula de ardilla con fallas en el rotor, en anillos y barras (Williamson et al., 1982), además, se empleó el monitoreo de vibraciones y corrientes para la detección de excentricidad en el entrehierro en motores de inducción de gran capacidad (Cameron et al., 1986). En los años noventa (Thomson et al., 1997) se presenta un método de monitoreo de la corriente en línea para el diagnóstico de excentricidad en el entrehierro, también se presentan resultados de diagnóstico en línea y fuera de línea de motores de inducción de baja mediana y alta tensión (Carvajal, et al., 1999). En el año 2000 (Fernández, et al., 2000) presentan una serie de técnicas de mantenimiento y diagnóstico de máquinas eléctricas rotatorias. Dentro de los trabajos reportados de una década hasta la actualidad, se tienen la aplicación de metodologías de diagnóstico de fallas en un motor de alta capacidad utilizado para una bomba de alta presión (Bossio, et al., 2005), posteriormente se presenta una metodología de monitoreo, detección y diagnóstico de fallas en motores asíncronos de inducción (Castelli, et al, 2007), y la aplicación de redes neuronales artificiales en el diagnóstico de fallas en motores de inducción (Villada, et al., 2007). Recientemente se han desarrollado nuevos libros dedicados al monitoreo de máquinas eléctricas rotatorias (Tavner et al., 2008), se han aplicado wavelets como una forma alternativa de detección de fallas (Schmitt et al., 2010, y se han presentado metodologías para la detección de fallas en rodamientos y engranajes (San Martín, et al., 2010).

\section{FALLAS MECÁNICAS EN MÁQUINAS ELÉCTRICAS ROTATORIAS}

Hay tres principales fallas mecánicas que se presentan en las máquinas eléctricas rotatorias, de las cuales una de ellas es el desequilibrio. Un sistema mecánico giratorio se dice que está equilibrado si durante su funcionamiento la resultante de todas las fuerzas y sus respectivos pares son de magnitud, dirección y sentido constantes. Lograda la constancia en módulo, dirección y sentido de las fuerzas, mediante una perfecta y homogénea distribución de las masas de la parte móvil, la máquina se puede sujetar mediante anclajes que opongan una fuerza y un momento de 
reacción a la resultante del sistema (Fernández et al., 2000). Dada la definición anterior de equilibrio, el desequilibrio se puede presentar de dos formas: una debida a una distribución no homogénea de masa que se puede detectar a rotor parado y otra asociada al movimiento del rotor.

Otra falla mecánica es el desalineamiento, el cual se debe a que es imposible que los ejes de la máquina eléctrica rotatoria bajo estudio y la máquina acoplada se encuentren perfectamente alineados en todos los planos (Fernández et al., 2000 y Albino, 2004). Finalmente, la última falla mecánica por mencionar es la falla de los rodamientos. Las máquinas rotatorias disponen de rodamientos sobre los que se apoyan los dos extremos del eje que sustenta a la parte móvil. Puesto que estos elementos del sistema están sometidos a una continua fricción y movimiento dan origen a las vibraciones causadas por cualquier defecto de la máquina o agente externo que se les transmite, por lo que son los componentes con un porcentaje de falla más elevado (Fernández et al., 2000 y Albino, 2004). Las fallas antes mencionadas producen deformaciones en el entrehierro, dando lugar al fenómeno conocido como excentricidad (Carvajal et al.,1999 y Albino, 2004,) . Existen dos tipos de excentricidad, los cuales se muestran en la Fig. 1 y se describen a continuación:

La excentricidad estática consiste en una distorsión en el tamaño del entrehierro en el cual el valor mínimo de este se encuentra en una posición fija en el espacio. Esta distorsión puede ser causada por la forma oval del estator o por un incorrecto posicionamiento del rotor dentro del estator, causado por un mal apoyo de los rodamientos, desgaste de éstos, malformación de los alojamientos, excesiva tolerancia, etc. (Fernández et al., 2000 y Torbar, 1998). En el caso de la excentricidad dinámica lo que ocurre es que el punto de entrehierro mínimo no permanece fijo en una posición en el espacio sino que gira solidario con el rotor. La causa de este comportamiento puede ser la forma oval del rotor, o el hecho de que el centro de giro de este último no sea su centro geométrico de rotación.

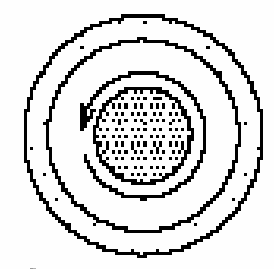

a)

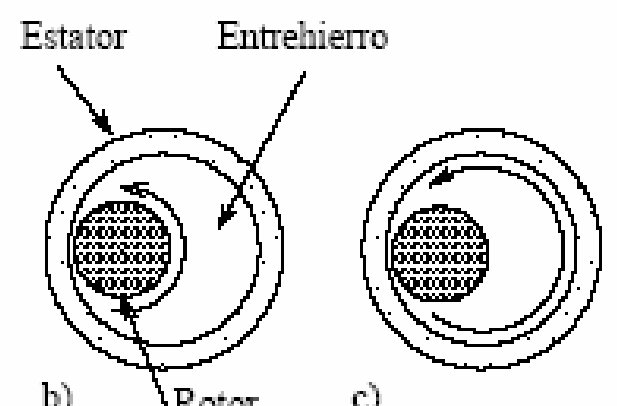

c)

Fig. 1: Formas en que se presenta la excentricidad. (a) Máquina sana, (b) excentricidad estática, (c) excentricidad dinámica

\section{FALLAS ELÉCTRICAS EN MÁQUINAS ELÉCTRICAS ROTATORIAS}

Esta parte se centra en las fallas eléctricas de los motores de inducción tipo jaula de ardilla, los cuales están construidos en su parte móvil (rotor) de barras de aluminio o cobre conforme a su potencia eléctrica. Estas están unidas en sus extremos por dos anillos en cortocircuito y al conjunto se le conoce como rotor jaula de ardilla. El núcleo del rotor esta conformado por acero magnético laminado; la jaula puede ser también construida de barras de cobre para el caso de motores de mediana y alta capacidad.

En el caso de barras rotas en motores de inducción tipo jaula de ardilla, para el proceso de visualizar y mostrar su influencia en el comportamiento del motor existen varios caminos de análisis y uno de ellos es mostrar un modelo con un circuito que considera la corriente de falla superpuesta a la corrientes circulantes por la jaula con todas las barras unidas a los anillos, es decir, considerar la distribución real de las corrientes (I) en la jaula como la superposición de dos casos: el primero es la distribución de corrientes por una jaula sana y el segundo caso consistirá 
en sustituir la barra rota por una fuente de corriente de igual valor y sentido contrario a la que circula por dicha barra en el primer caso. A ambos circuitos se les aplica el teorema de superposición con lo cual se logra que la corriente en la barra rota quede anulada (Fernández et al., 2000). Esto se muestra en la Fig. 2 siguiente:

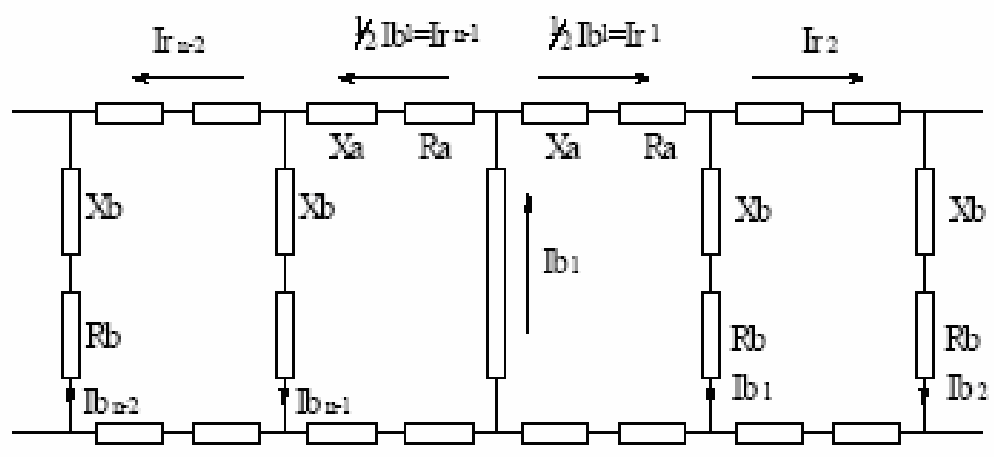

\author{
$\mathrm{Ra}=$ Resistercia del anillo de carto circuito \\ $\mathrm{Xa}=$ Reactancia del anillo de carto circuito \\ $\mathrm{Rb}=$ Resistencia de la berra \\ $\mathrm{X} b=$ Reactamia de la beura \\ Ibl=Coriente inyectacka en la brora rota
}

Fig. 2: Circuito equivalente al segundo término (barra rota en la posición 1)

En casos de detección de fallas en el rotor se tiene que los devanados de una máquina sana no son ideales y se considera la existencia de armónicos espaciales en el estator y en el rotor, los cuales son originados por el campo B compuesto por la suma de dos campos senoidales de eje fijo que pulsaran en el tiempo a la frecuencia de deslizamiento, a estos se les aplica el teorema de Leblanc(Fernández et al., 2000) y se tendrá que ambos giran en sentidos opuestos con respecto al rotor a la velocidad de deslizamiento. Estos campos están formados por una serie de armónicos espaciales y aparecerán superpuestos a los ya existentes en el motor.

Esto es lo que se llama en el diagnóstico de fallas en el rotor, el análisis espectral de las corrientes de alimentación, donde se observa cuales son las frecuencias que inducen los armónicos de campo debidos por la falla en las corrientes del rotor, y para esto hay que tomar en cuenta los factores de diseño de los devanados de estator que afectan las frecuencias inducidas en las corrientes ya que algunos de los armónicos causados por la falla no podrán inducir fuerzas electromotrices, aunque solo se toman en cuenta las frecuencias inducidas por un armónico general y así tomar todos los casos posibles y posteriormente se comparan los resultados obtenidos con los armónicos en el terreno práctico con los espectros de frecuencia de la corriente de los motores con asimetría en el rotor (Fernández et al., 2000 y Tavner et al., 2008) . Esta metodología consiste en calcular la velocidad a la que se mueven los armónicos sobre el estator, siendo esta para el armónico fundamental:

Ns-N

$\mathrm{Ns}=\mathrm{f} / \mathrm{P}$

\footnotetext{
Ns = Velocidad de sincronismo $(\mathrm{rpm} / \mathrm{s})$

$\mathrm{N} \quad=$ Velocidad de giro del rotor $(\mathrm{rpm} / \mathrm{s})$

$\mathrm{f} \quad=$ Frecuencia de operación de la máquina $(\mathrm{Hz})$

$\mathrm{P} \quad=$ Número de pares de polos
}

Por consiguiente la velocidad eléctrica con respecto al rotor del armónico fundamental en el sentido de la serie será: 
En forma general se tiene: $P(N s-N) / i$. que referido al estator es: $P(N s-N) / i+N=P N s / i+N(1-$ $\mathrm{P} / \mathrm{i})$, finalmente se tiene: $\mathrm{P} N \mathrm{~N}+\mathrm{N}(\mathrm{i}-\mathrm{P})$ causado por el armónico debido a la falla de fractura de la barra. Por último las frecuencias inducidas en el estator por el armónico de falla son:

$f+N(i-P)=f[i / P(1-S)+S]$

$-f+N(i+P)=f[i / P(1-S)-S]$

donde S es el deslizamiento, e i el orden del armónico.

En el caso de presencia de excentricidades estáticas y dinámicas, estas dan como resultado picos de componentes de corriente en las frecuencias dadas por la siguiente expresión (Carvajal, et al., 1999)

$$
f=f 1[(k R \pm n d)(1-S) / P \pm n w]
$$

f1 =es la frecuencia de línea del motor

$\mathrm{R} \quad=$ número de ranuras del rotor

k $=1,2,3,4,5, \ldots$

nw $=1,3,5,7,9, \ldots$

nd $\quad=0$, para excentricidad estática

nd $\quad=1,2,3,4,5 \ldots$ para excentricidad dinámica

Otra forma de detectar la presencia de la excentricidad combinada es analizando las frecuencias originadas por el giro del rotor en Hertz, respecto del estator, es decir f1+fr.

\section{DIAGNÓSTICO DE MÁQUINAS ELÉCTRICAS ROTATORIAS EMPLEANDO LA FFT}

En las máquinas rotatorias, cualquiera de los dos tipos de excentricidad los efectos producidos sobre su funcionamiento son los mismos (Fernández et al., 2000):

1. Aparición de armónicos de alta y baja frecuencia en el espectro de las corrientes del estator.

2. Aparición de fuerzas pulsantes de origen eléctrico que darán lugar a vibraciones características.

3. Incremento general de la vibración de la máquina.

El análisis en el dominio de la frecuencia de la señal de las corrientes de la máquina refleja claramente los efectos de la excentricidad, por el hecho de que este análisis permite detectar variaciones en la señal de muy pequeña amplitud (Fernández et al., 2000). Para dicho análisis en el dominio de la frecuencia se emplea la FFT que es un algoritmo para el cálculo aproximado de la transformada discreta de Fourier.

Esta técnica se ha empleado desde hace varios años en el diagnóstico de motores para detectar fallas en el rotor mediante el análisis de de las corrientes en el dominio de la frecuencia y los armónicos mas analizados son los situados al lado de de la frecuencia fundamental. A estas frecuencias se les llama armónico lateral inferior fi y superior fs ,y sus magnitudes están en función del grado de asimetría que presenta el rotor tipo jaula de ardilla (Fernández et al., 2000 y Thomson et al., 2001). Esto se puede observar en la Fig. 3.

\section{DESCRIPCIÓN DE LOS SISTEMAS DE PRUEBA}

En el caso de desalineamiento se tiene un grupo de motor de corriente directa (7.5 HP)-generador síncrono (5kVA, $220 \mathrm{~V}, 60 \mathrm{~Hz}, 13.5 \mathrm{~A})$, los cuales presentan esta falla mecánica, este arreglo se muestra en las Figs. 4 y 5. 


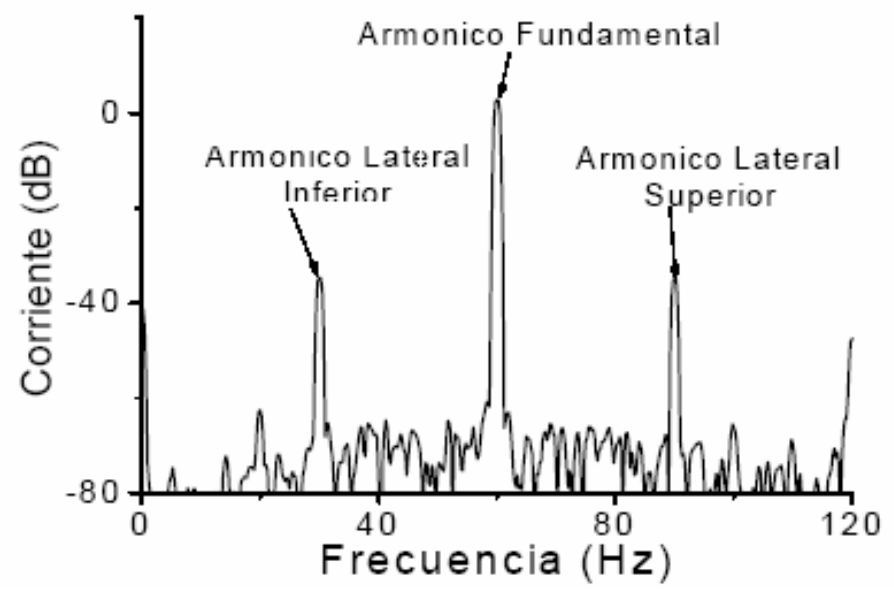

Fig. 3: Armónicos lateral inferior y lateral superior de las corrientes

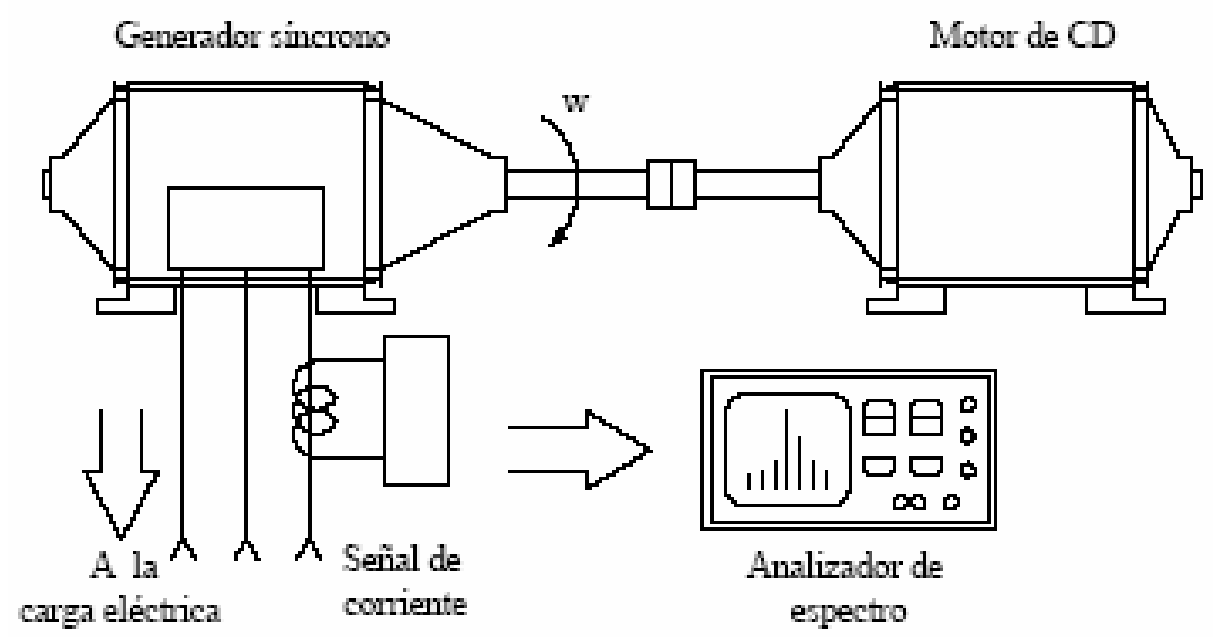

Fig. 4: Sistema de prueba para desalineamiento del acoplamiento del motor de CD y el generador síncrono

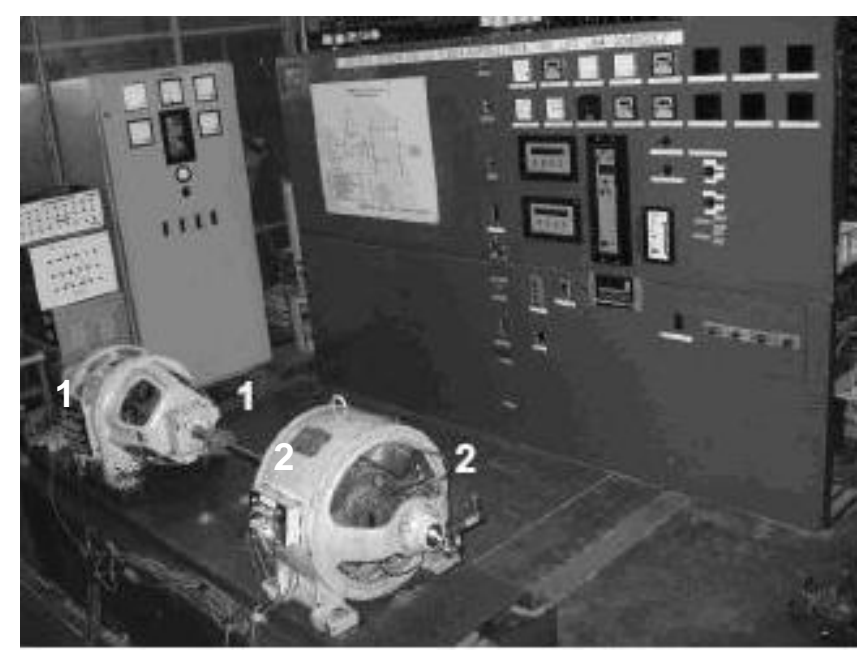

Fig. 5: 1) Generador síncrono de polos salientes bajo prueba, 2) Motor de CD de 7.5 HP 
El motor de inducción empleado (tipo jaula de ardilla de $5 \mathrm{HP}$ a $220 \mathrm{~V}, 60 \mathrm{~Hz}$ ) presenta una falla de holgura de baleros. El sistema de prueba se muestra en las Figs. 6 y 7 , donde el arreglo de prueba es mediante generador de C.D y carga resistiva variable

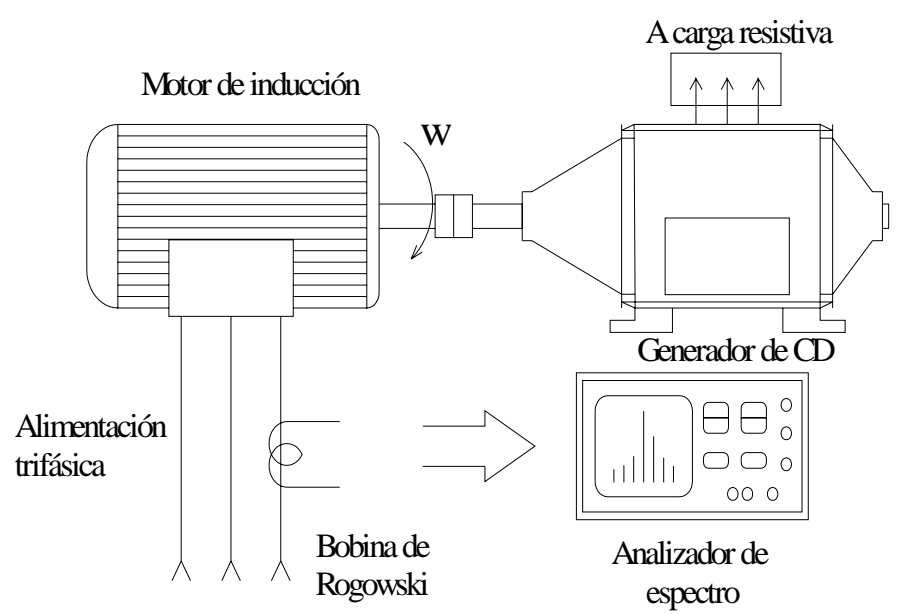

Fig. 6: Sistema de prueba para el motor de inducción jaula de ardilla

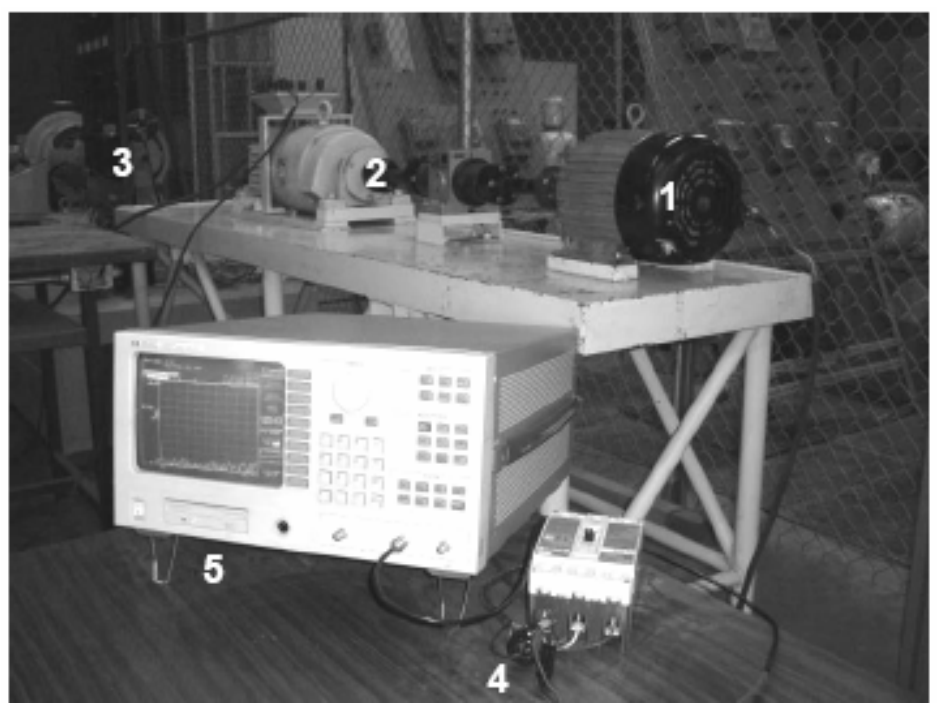

Fig. 7: Sistema físico de prueba. 1) Motor de inducción, 2) Generador de CD, 3) Carga resistiva, 4) Bobina de Rogowski, 5) Analizador de espectros

\section{RESULTADOS EXPERIMENTALES}

Para la aplicación de la metodología se emplearon máquinas eléctricas rotatorias de laboratorio. El grupo motor de CD-generador síncrono se desalineó con el propósito de obtener mediciones en dichas condiciones para después compararlas, después de haber alineado el sistema. En el caso del motor de inducción, se adaptaron dos prototipos con las mismas características eléctricas de operación nominales; al primero se le desgastaron los soportes de los rodamientos para provocar la holgura de los mismos, al segundo se le seccionaron dos barras de la jaula. En el caso del grupo motor de CD- generador síncrono este es de polos salientes con tres pares de polos y 54 ranuras; los motores de inducción cuentan con 36 ranuras en el estator y 48 barras en la jaula.

En el caso del generador síncrono se presentan resultados debidos al desalineamiento del grupo motor de CD-generador síncrono para diferentes porcentajes de su carga nominal y sin carga. Las cargas que se emplearon básicamente son resistivas e inductivas y la combinación de las mismas. Las gráficas para este caso se muestran en las Figs. 8, 9 y 10 respectivamente. 


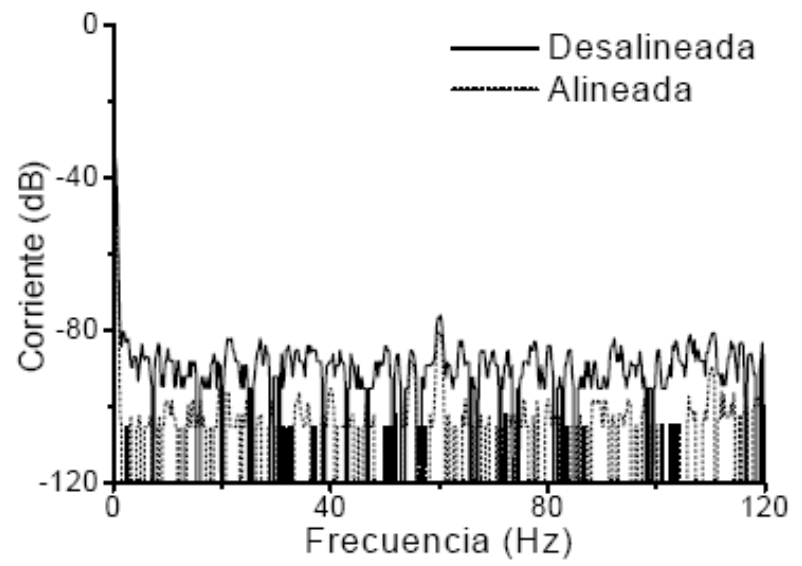

a)

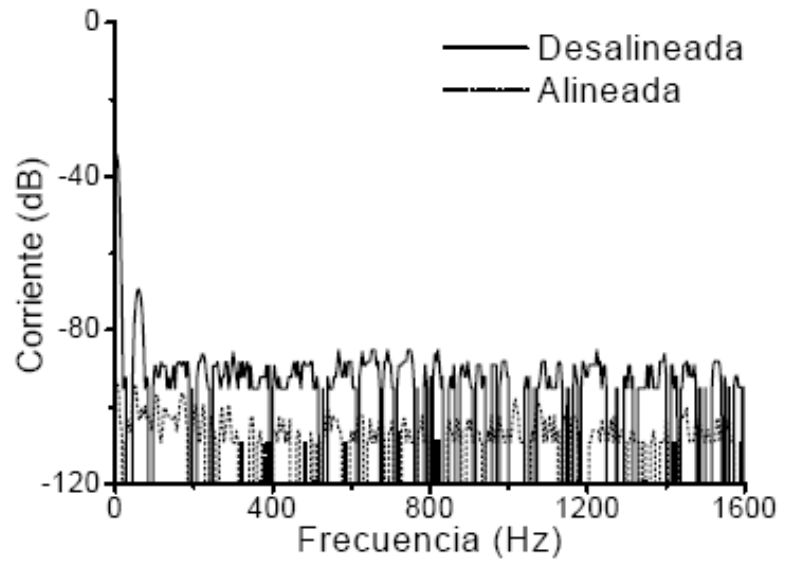

b)

Fig. 8: Comparación de los espectros de corriente con generador sin carga, desalineado y alineado, para diferentes barridos de frecuencia. (a) 0-120 Hz, (b) 0-1600 Hz

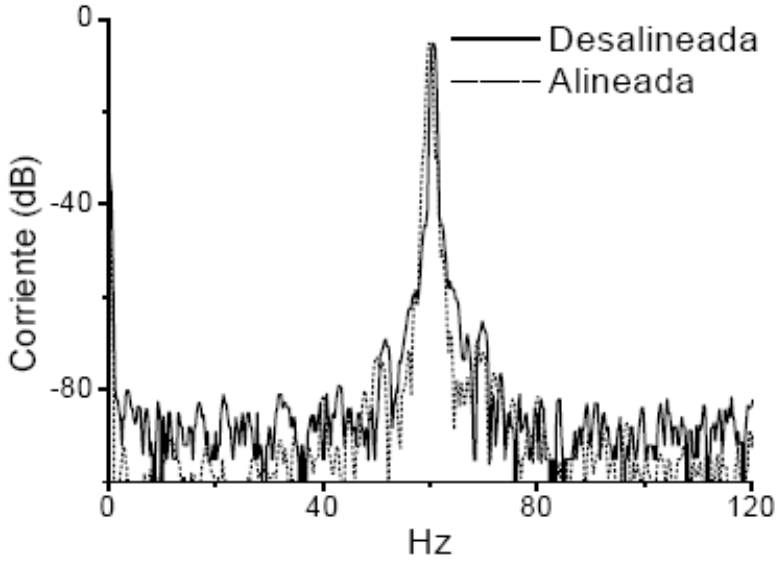

a)

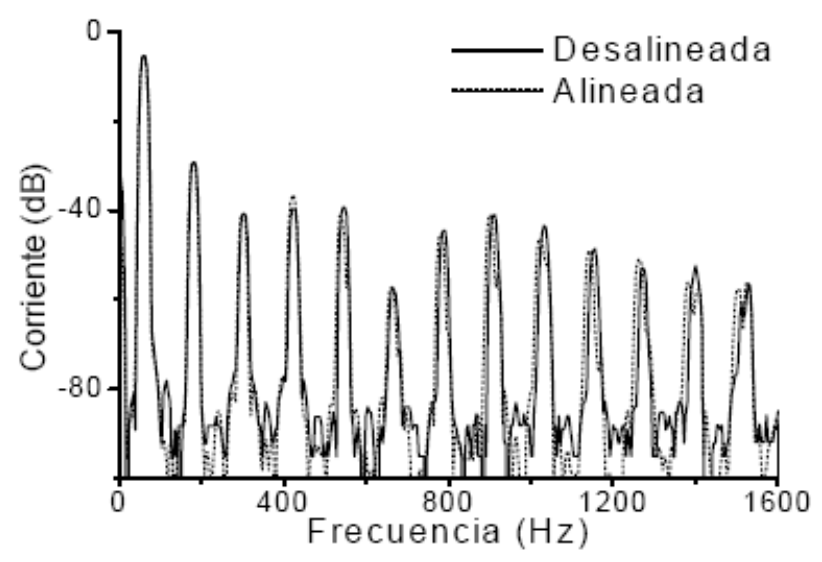

b)

Fig. 9: Comparación de los espectros de corriente con generador con $50 \%$ de su carga nominal, desalineado y alineado, para diferentes barridos de frecuencia. (a) 0-120 Hz., (b) 0-1600 Hz.

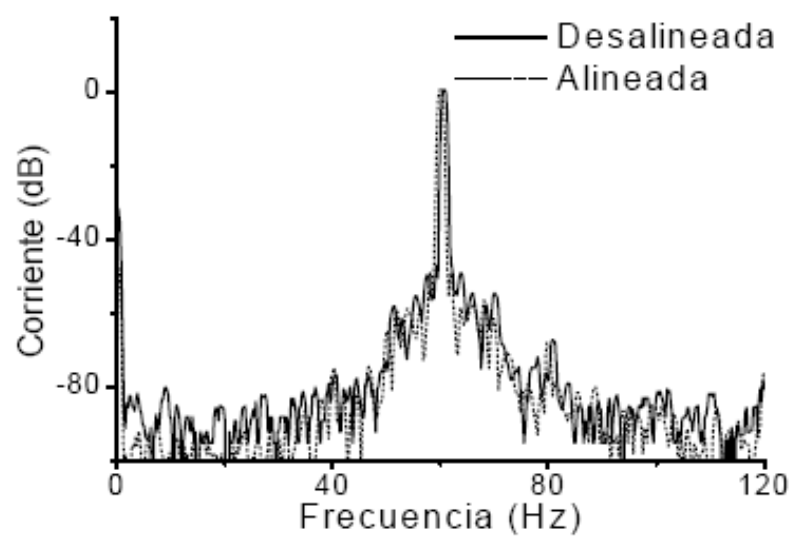

a)

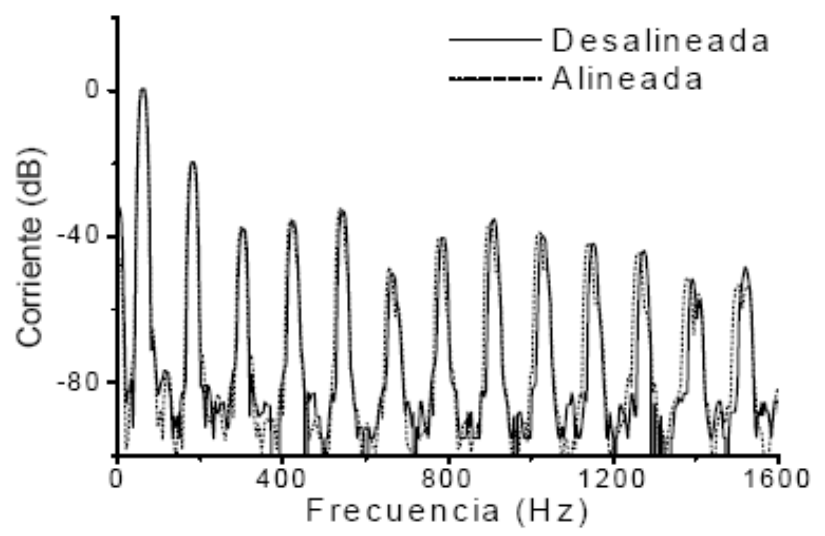

b)

Fig. 10: Comparación de los espectros de corriente con generador con 100\% de su carga nominal, desalineado y alineado, para diferentes barridos de frecuencia. (a) 0-120 Hz, (b) 0-1600 Hz 
Los resultados de las pruebas realizadas al motor de inducción en vacío y con carga, con holgura en soporte de baleros, se presentan en la siguiente Fig.11 a y b.

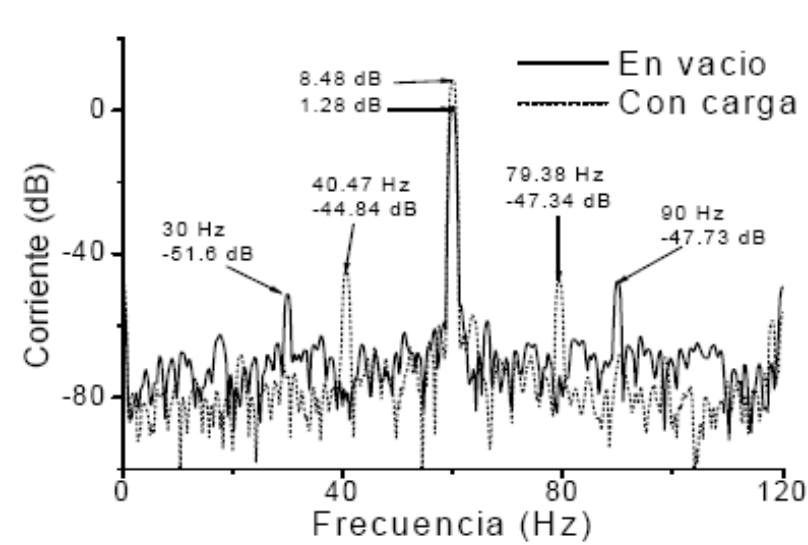

a)

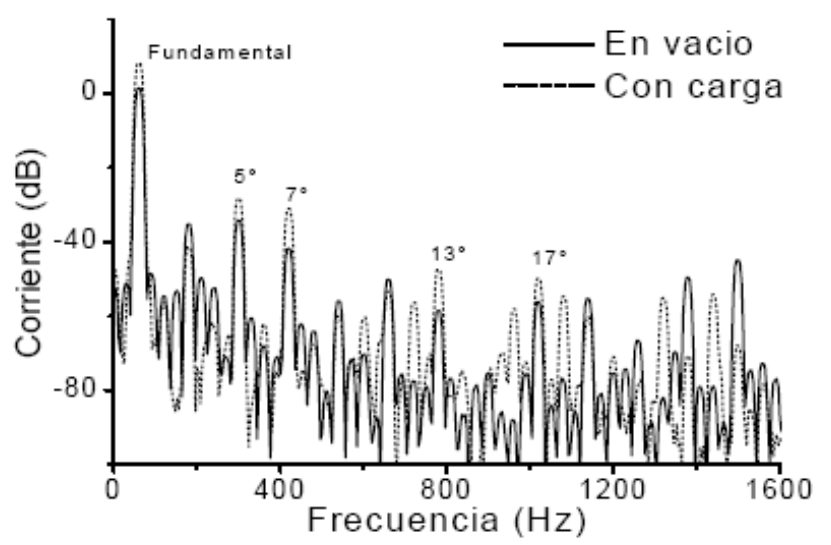

b)

Fig. 11: Comparación de espectros de corriente del motor experimental con holgura en soporte de baleros, con carga y sin carga, para diferentes barridos de frecuencia. (a) $0-120 \mathrm{~Hz}$, (b) $0-1600 \mathrm{~Hz}$

De los espectros anteriores se puede apreciar que al aumentar la carga del motor, aumentan las magnitudes en $\mathrm{dB}$ de las frecuencias de las bandas laterales. En el caso del motor con carga existe una diferencia de aproximadamente $55.8 \mathrm{~dB}$ entre la magnitud de la fundamental y la frecuencia $f_{-} f r$, correspondiente a las frecuencias que aparecen en presencia de excentricidad combinada, esto debido a la holgura de los soportes de baleros del motor. Además, en el espectro de mayor frecuencia se aprecia un incremento en las frecuencias de los armónicos impares de la fundamental.

Se realizaron mediciones con carga para un motor de 5HP, con falla de dos barras rotas en el rotor y midiendo la corriente con bobina de Rogowski (Manufacturer Bergoz Instrumentation). En las mediciones mostradas en las figuras anteriores se empleo una convencional. En la Figura 12 a) y b) se puede observar que las mediciones obtenidas son aproximadas. En la Figura 13 a y $b$ se muestran los espectros de un motor sano y fallado y medido con ambas bobinas.

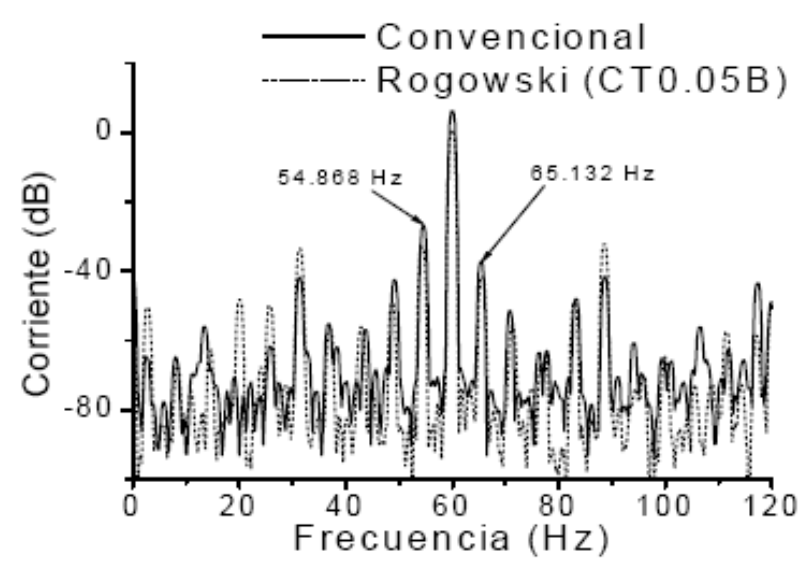

a)

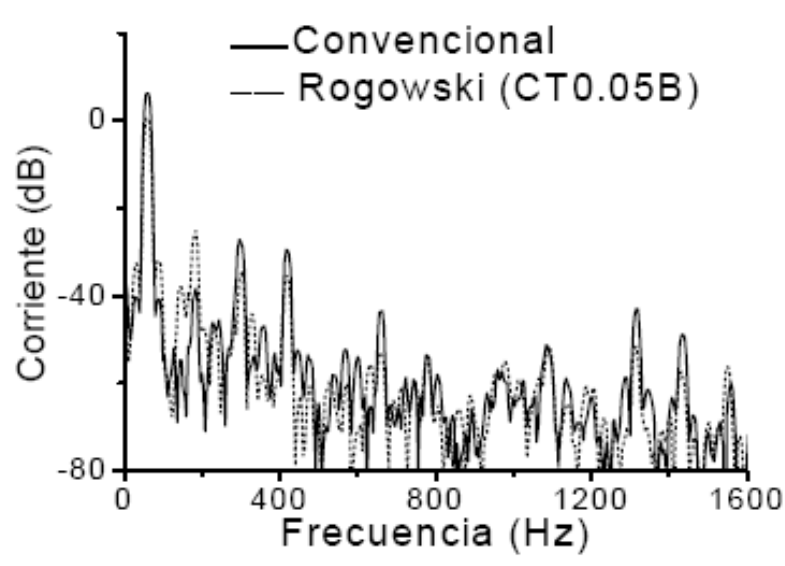

b)

Fig. 12: Comparación de los espectros de corriente del motor experimental con falla de barras rotas, con carga nominal, para diferentes barridos de frecuencia empleando una bobina de Rogowski (a) 0-120 Hz, (b) 0-1600 Hz 
En este caso aparecen las bandas laterales correspondientes a las frecuencias $f 1(1 \pm 2 S)$ debido a la falla en las barras. Las magnitudes entre la frecuencia fundamental y las bandas laterales es de $33 \mathrm{~dB}$, lo cual indica que existe mas de una barra rota en la jaula. Sin embargo, en el espectro a $1.6 \mathrm{kHz}$ existe la presencia de armónicos, pero estos se pueden presentar por la distribución del devanado.

(a

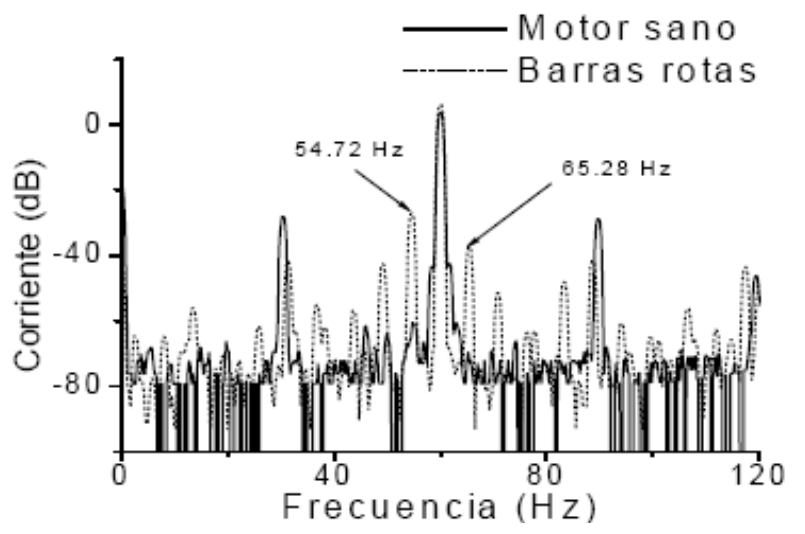

(b)

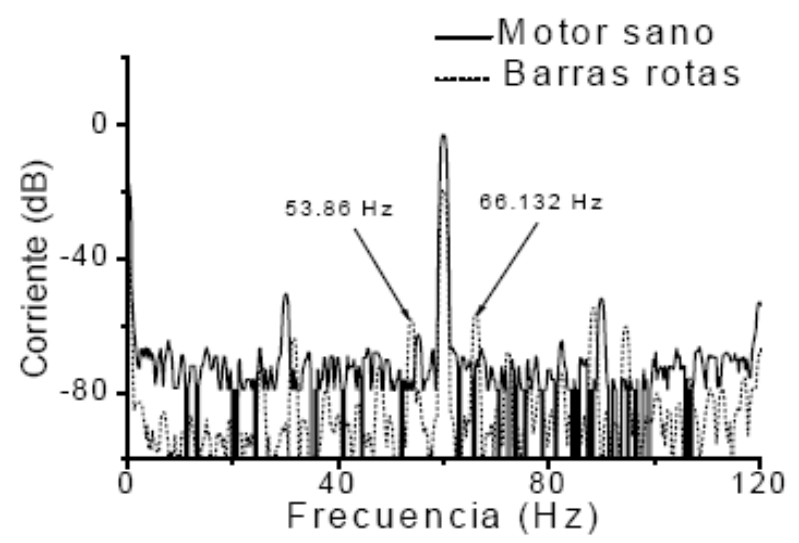

Figura 13: Comparación de resultados de un motor sano y uno con barras rotas, con carga nominal. (a) bobina convencional, (b) bobina de Rogowski

En los espectros de la Fig. 13 se observa que aumentan los valores en $\mathrm{dB}$ correspondientes a las frecuencias de falla de barras teniendo una diferencia con la fundamental de $33.24 \mathrm{~dB}$ y $36.5 \mathrm{~dB}$ para el caso a y b respectivamente, a diferencia del caso sano que se tiene $64.15 \mathrm{~dB}$ y $67.91 \mathrm{~dB}$, esto nos indica que existen varias barras rotas. Las diferencias anteriores para un motor sano se encuentran entre los rangos de $54 \mathrm{~dB}$ a $60 \mathrm{~dB}$ y para un caso de operación aceptable de $48 \mathrm{~dB}$ a 54 dB (Fernández et al., 2000).

\section{CONCLUSIONES}

La técnica presentada en el dominio de la frecuencia para diagnóstico de fallas no es destructiva, se realiza en línea. El espectro de las bandas laterales de frecuencias cercanas a la frecuencia de 60 Hertz se presenta para el caso del desalineamiento en mayor magnitud, con carga que en vacío, del grupo motor de C.D.-generador síncrono, así también para el caso de holgura de soportes de baleros del motor. Adicionalmente se presentan resultados de fallas por barras rotas y motor sin fallas, comparando la bobina de Rogowski contra la bobina convencional, observándose que la diferencia entre ellas es debido al ancho de banda con el que fueron diseñadas. No obstante, las frecuencias laterales objeto de estudio, pueden ser analizadas con ambas bobinas, ya que las magnitudes obtenidas de las señales son adecuadas para realizar la medición del espectro de la corriente. A pesar de que cada falla presenta frecuencias laterales que las distingue, se pueden presentar frecuencias armónicas que no necesariamente sean indicio de 
falla, estas son originadas también por los armónicos espaciales debidos a la distribución de los devanados de la máquina.

\section{AGRADECIMIENTOS}

Agradecemos a la empresa Ingeniería y Diseño Electromecánico, S.A. de C.V. (IDEMSA) por el apoyo brindado tanto en la preparación del prototipo del motor de inducción como en la alineación del grupo generador síncrono-motor de CD.

\section{REFERENCIAS}

Albino, P. I. , Impacto de la Excentricidad Estática en la Estabilidad Transitoria de un Generador de Polos Salientes, tesis de maestría, Programa de Maestría en Ciencias en Ingeniería Eléctrica, SEPI-ESIME-IPN, D.F., México (2004).

Bossio, G.R., De Angelo, C. H., De Pauli, A. D., García, G. O., Diagnóstico de fallas en el rotor de motores de inducción caso de estudio: motor de 1.6 MW de bomba de alta presión, XI Reunión de Trabajo en Procedimiento de la Información y Control, 21 al 23 de Septiembre (2005).

Carvajal, F. A., Ramírez, J. M., Arcos, L. F., Diagnóstico en línea y fuera de línea de motores de inducción de baja, mediana y alta tensión, Boletín IIE, Marzo-Abril, México (1999).

Castelli, M., Andrade, M., Metodología de monitoreo, detección y diagnóstico de fallos en motores asíncronos de inducción, URUMAN, Montevideo-Uruguay (2007).

Fernández, C. M., García, M. M., Alonso, O G., Cano, R. J.M., Solares, S. J., Técnicas para el mantenimiento y diagnostico de máquinas eléctricas rotativas, ABB Service S.A.-Marcombo Boixareu Editores, España (2000).

Fitzgerald, A. E., Kingsley, C. Jr., Umans, S. D., Máquinas eléctricas, sexta edición.,. McGraw-Hill, México (2004).

Manufacturer Bergoz Instrumentation,Espace Ollondon Ouest,01630 Saint Genis Pouilly, France.

San Martín, C., Estupiñán, E., San Martín, D., A methodology for the detection and diagnostic of localized faults in gears and rolling bearing systems, Ingeniare. Revista chilena de ingeniería, 18(1), 44-52 (2010).

Schmitt, E, Idowu, P., Morales A., Applications of wavelets in induction machine fault detection, Ingeniare. Revista chilena de ingeniería, 18(2), 158-164 (2010).

Tavner, M., Ran, L., Penman, J., Sennding, H., Condition Monitoring Of Rotating Electrical Machines, IET Power And Energy Series 56, Reino Unido (2008).

Thomson, W. T., Rankin, D., Dorrel, D. G., On-line current monitoring to diagnose airgap eccentricity - an industrial case history of a large high-voltage three-phase induction motor, IEEE, MA2-4.1- MA2-4.3 (1997).

Thomson, W.T., Fenger, M., Current signature analysis to detect induction motor faults. IEEE Industry Applications Magazine, 26-34, (2001).

Torbar, T. W., Online current monitoring and application of a finite element method to predict the level of static airgap eccentricity in three-phase induction Motors, IEEE Trans. On energy conversion, vol. 14, no.4, pp 347-357, (1998).

Villada, F.,Cadavid, D. R., Diagnóstico de fallas en motores de inducción mediante la aplicación de redes neuronales artificiales, Información Tecnológica,18(2), 105-112, Chile (2007).

Williamson, S., Smith, A. C., Steady-state analysis of a 3-phase cage motors with rotor-bar and end ring fault, IEE PROC, 129(3), 93-100 (1982). 
